\title{
Antecedentes da intenção empreendedora: uma perspectiva com alunos da pós graduação
}

Background of the Understanding Entrepreneur: a perspective with postgraduate students

\section{Denise Adriana Johann', Ricardo Alberti", Andrieli de Fátima Paz Nunes"', Luis Felipe Dias Lopesiv, Fernando de Jesus Moreira Juniorv}

\begin{abstract}
RESUMO
A intenção empreendedora é definida como uma característica pessoal que antecede um comportamento, podendo ser um estado de espírito onde o indivíduo está dirigido para algo que deseja alcançar ou ser formada momentos antes da concretização de uma ideia. Conhecer a intenção empreendedora, as diferenças e semelhanças entre as atitudes dos indivíduos podem nos levar as razões que os levam a empreender. O objetivo da pesquisa foi analisar a intenção empreendedora nas dimensões de atitudes pessoais, normas subjetivas e percepção de controle de planejamento, com base em uma amostra de 107 alunos de pós-graduação da Universidade Federal de Santa Maria. O método de pesquisa possui natureza aplicada, com abordagem quantitativa, com objetivo exploratório e descritivo. Foi utilizado o levantamento técnico de dados por survey, utilizando modelagem de equações estruturais, a partir de três hipóteses, onde somente a hipótese H1 (Atitudes Pessoais se relacionam direta e positivamente com a Percepção de Controle de Planejamento) foi aceita, H2 (Normas Subjetivas se relacionam direta e positivamente com a Percepção de Controle de Planejamento) e H3 (Normas Subjetivas se relacionam direta e positivamente com as Atitudes Pessoais) foram rejeitadas.
\end{abstract}

Palavras-chave: Intenção Empreendedora; Pós Graduação; Equações Estruturais.

\begin{abstract}
Entrepreneurial intent is defined as a personal trait that precedes a behavior and can be a state of mind in which the individual is directed toward something he wishes to achieve or to form moments before the realization of an idea. Knowing the entrepreneurial intent, differences and similarities between the attitudes of individuals can lead us to the reasons that lead them to undertake. The objective of this research was to analyze the entrepreneurial intention in the dimensions of personal attitudes, subjective norms and perception of planning control, based on a sample of 107 postgraduate students from the Federal University of Santa Maria. The research method is applied in nature, with quantitative approach, with exploratory and descriptive objectives. The technical survey of data was used using structural equation modeling from three hypotheses, where only hypothesis $\mathrm{H} 1$ (Personal Attitudes are directly and positively related to Planning Control Perception) was confirmed, $\mathrm{H} 2$ (Subjective Norms if directly and positively related to Planning Control Perception) and H3 (Subjective Norms directly and positively related to Personal Attitudes) were rejected.
\end{abstract}

Keywords: Entrepreneurial Intention; Postgraduate; Structural equations. 


\section{INTRODUÇÃO}

O empreendedorismo está vinculado ao comportamento e atitude empreendedora no sentido de despertar nas pessoas o desejo de criação de novos negócios, produtos ou criação de valor em algo já existente. Há diferentes perspectivas e abordagens que buscam explicar ou demonstrar de que forma o construto intenção empreendedora afeta a atitude de empreender. A importância de estudos na perspectiva da intenção empreendedora (IE) tem se mostrado relevante, tanto no contexto acadêmico como no mundo dos negócios, já que as intenções são utilizadas para capturar os fatores motivacionais que influenciam o comportamento e são indicadores do quanto as pessoas estão dispostas a tentar ou se esforçar para executar um comportamento (AJZEN, 1991).

Nossa intenção em fazer algo, através de nossas atitudes se materializa pelo nosso comportamento referente a algo. A atitude empreendedora é explicada pela Teoria do Comportamento Planejado de Ajzen (1985), que define isso como uma avaliação de um objeto de estímulo, influenciado por crenças. Bird (1988) pontua que com o propósito de alcançar uma meta, a intenção pode ser identificada como um estado de espírito em que a atenção e os objetivos do indivíduo estão focados para determinada situação.

Através da intenção empreendedora é possível entender o processo de empreendedorismo em um âmbito geral. As intenções são consideradas fundamentais para o estudo do comportamento humano (ARAÚJO, 2014). Para o autor, a intenção empreendedora pode ser identificada por uma vontade própria que uma pessoa possui de abrir um novo negócio. Pode ser algo concreto ou que nunca virá a acontecer. É algo que há potencial, mas que precisa ser despertado, desenvolvido e aperfeiçoado para que aconteça. Segundo Mueller, Zapkau e Schwens (2014), a intenção empreendedora nasce a partir do comportamento empreendedor, em que o indivíduo relaciona o compromisso e a intenção em ter o seu negócio.

Segundo Araújo (2014) os fatores externos são influenciadores e estão associados com as atitudes de um indivíduo em relação a se tornar ou não um empreendedor. Sabe-se que as intenções e atitudes variam de uma pessoa para outra. 
Por este motivo, existem fatores que são influenciadores na intenção de empreender. Dentre os principais, podemos destacar as crenças, os hábitos, os valores pessoais, os desejos, as necessidades e as oportunidades que o sujeito se encontra.

Liñan e Chen (2009) desenvolveram com base em Ajzen (1991), o questionário de intenção empreendedora (QIE), que tem o objetivo de testar o modelo de intenção empreendedora, medindo a referida intenção e as variáveis que a influenciam. As variáveis determinantes da intenção empreendedora podem ser descritas, como atitude pessoal que refere-se à impressão positiva ou negativa que o indivíduo tem sobre ser empreendedor; a norma subjetiva que refere-se a pressão social exercida sobre o indivíduo para tornar-se ou não um empreendedor, proveniente do círculo social em que vive; e, controle comportamental percebido que refere-se à percepção do indivíduo sobre a facilidade ou dificuldade de se tornar um empreendedor e sua capacidade em empreender.

As atitudes pessoais conforme Ajzen (2001) são elencadas à atitude e crença do indivíduo em relação a um comportamento, ou seja, qual a percepção do indivíduo sobre a possibilidade de se tornar um empreendedor. Enquanto as normas subjetivas referem-se à percepção do indivíduo quanto à pressão social exercida sobre ela no contexto de ser empreendedor, e a opinião do grupo de referência na sua decisão de se tornar um empreendedor (AJZEN, 2001). Já, a percepção de controle de comportamento refere-se à própria percepção do indivíduo sobre a manifestação do comportamento empreendedor.

Poucos estudos são encontrados na literatura que analisam a relação das normas subjetivas com as atitudes e com o controle comportamental percebido. Os estudos em destaque são os de Liñán e Chen (2009) os quais são utilizados como referência no modelo aplicado no presente artigo. Liñán e Chen (2009) obtiveram entre os resultados do seu estudo que a principal influência das normas subjetivas foi exercida através de seus efeitos na atitude pessoal e no controle do comportamento percebido, obtendo caminhos significativos para as respectivas hipóteses testadas. Podendo haver razões, portanto, para considerar que as normas subjetivas possuem efeito tanto na atitude pessoal quanto no controle comportamental percebido. Tal 
modelo é exposto na Figura 1: Destas discussões emergem as três hipóteses deste estudo:

$\mathrm{H}_{1}$ : Atitudes Pessoais se relacionam direta e positivamente com a Percepção de Controle de Planejamento;

$\mathrm{H}_{2}$ : Normas Subjetivas se relacionam direta e positivamente com a Percepção de Controle de Planejamento;

$\mathrm{H}_{3}$ : Normas Subjetivas se relacionam direta e positivamente com as Atitudes Pessoais;

Figura 1 - Modelo de mediação das dimensões da escala de Intenção Empreendedora

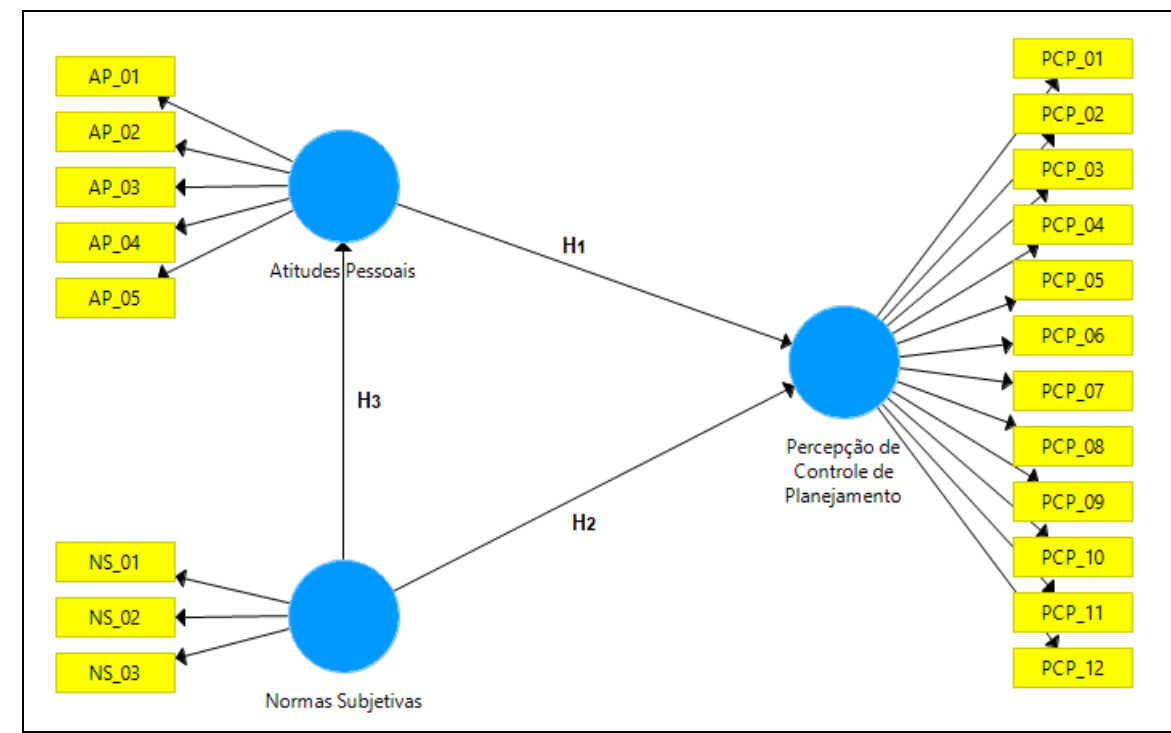

Fonte: Desenvolvido pelos autores (Software Smart PLS® v. 3.0 (RINGLE; WENDE; BECKER, 2015).

Liñan e Chen (2009) explicam que além destas variáveis, o modelo de intenção empreendedora destaca também o capital humano que são as habilidades, capacidades e destrezas individuais que os indivíduos possuem e desenvolvem com o intuito de crescimento profissional e pessoal; e outras variáveis demográficas que influenciam indiretamente na formação da intenção empreendedora, como a experiência e a educação do indivíduo.

Neste sentido, Davidsson (1995) defende que intenção empreendedora pode ser prever, embora de forma imperfeita, determinado comportamento de um indivíduo em relação a fundar a sua própria empresa. Araújo (2014) corrobora esta informação, já que relaciona a intenção empreendedora com a representação de um objetivo que a pessoa almeja alcançar e também, como o plano de ação que será fundamental na busca pela realização do objetivo a ser alcançado. Além disso, perceber as boas oportunidades, possuir conhecimentos, habilidades e experiências para começar um 
novo empreendimento, podem ser fundamentais para transformar a intenção empreendedora em um ato empreendedor, ou seja, colocá-la em ação.

\section{REFERENCIAL TEÓRICO}

Diversos autores ao longo do tempo têm estudado e contribuído para a definição de intenção empreendedora Carter, Gartner e Reynolds (1996) em seu estudo analisou o período de criação correlacionando o tempo de intenção e a ação propriamente dita. Neste estudo os autores descobriram a inspiração como intenção empreendedora confirmando uma ligação intencional e testaram sobe ela a educação com uma influência exógena neste contexto com a Teoria do Comportamento Planejado.

Ajzen (1991) considera que o indivíduo avalia sua atitude ou crença de forma positiva ou negativa agindo de forma direta sobre seu comportamento variando desta forma sua intenção sob sua ótica do grau de dificuldade ou facilidade no que deseja realizar ou alcançar. Já para Handrimurtjahjo, Setiadi e Kuncoro (2015) a partir de seus estudos afirmam que os estudantes estão cientes da atitude e intenção empreendedoras como fatores determinantes do sucesso empresarial, com ligação direta nas competências e qualidades, entendendo que atitudes empreendedoras levam a construção de intenções.

Ajzen (1991), através do princípio geral da teoria psicológica do comportamento planejado, considera que ao abrir um negócio o indivíduo esta tendo um comportamento planejado, estes comportamentos são intencionais, definindo que há intenção para este comportamento, a intenção é prevista por atitude .

Nessa perspectiva Loiola et al. (2016) definem a intenção empreendedora como uma projeção pessoal, a qual sustenta o empreendedorismo ser consequência da intenção empreendedora baseado no desejo, na análise de oportunidades e a propensão para agir nessa direção e o segundo modelo: da ação planejada. Duas teorias também norteiam o construto intenção empreendedora, a Teoria da ação racional (FISHBEIN; AJZEN, 1975) onde as atitudes individuais e as normas subjetivas atuam como antecedentes da intenção comportamental e a Teoria da ação planejada (AJZEN, 1985) que acredita o indivíduo ter a percepção e o controle sobre seu comportamento identificando e calculando riscos (LOIOLA et al., 2016). 
Neste contexto Linan, Urbano e Guerrero (2011) acreditam o incentivo ao desenvolvimento de intenções empreendedoras em estudantes através da educação para o empreendedorismo com o objetivo de estimular o comportamento empreendedor com foco na aprendizagem empreendedora e inspiração serem elementos chaves para Educação empreendedora. Desta forma o processo da educação empreendedora se dá de duas formas, através da aprendizagem empreendedora elencada ao conhecimento e as habilidades e através da inspiração aliada a motivação direcionando o comportamento com a transformação da mente e do coração a alcançar o que deseja (LINAN; URBANO; GUERRERO, 2011).

Em virtude disso torna-se relevante investigar a intenção em empreender bem como os fatores que a influenciam de forma acreditar-se o estudo das intenções empreendedoras uma maneira de promover a pesquisa sobre empreendedorismo.

\section{MÉTODO DE PESQUISA}

Quanto ao método, a pesquisa é caracterizada por abordagem quantitativa (HAIR JR. et al., 2014), objetivo exploratória (GIL, 1996) e descritiva (APPOLINÁRIO, 2011). Para Gil (2010) a pesquisa exploratória tem como objetivo proporcionar maior conhecimento do problema. Na etapa exploratória foram aplicadas técnicas estatísticas multivariadas de análise fatorial, utilizada para descrever e interpretar os resultados.

Na etapa descritiva, o objetivo foi mensurar a amostra da pesquisa para fins de caracterização. A pesquisa utilizou como procedimento técnico de levantamento, o método survey de coleta dedados. Por meio de um instrumento de pesquisa, é coletado sistematicamente um conjunto de dados quantificáveis, a respeito de um número de variáveis, que são então examinadas para discernir padrões de associação. Por esta razão, foi desenvolvido um instrumento estruturado de coleta de dados, aplicado junto aos alunos de Graduação e Pós-Graduação da Universidade Federal de Santa Maria, no Rio Grande do Sul.

O instrumento de pesquisa foi formado por questões compondo as três dimensões referentes ao conceito de intenção empreendedora de Liñán e Chen (2009), envolvendo 20 variáveis, sendo dividas conforme as dimensões da intenção 
empreendedora, segundo Liñán e Chen (2009). Assim, foram estruturadas: atitudes pessoais (cinco variáveis), normas subjetivas (três variáveis) e percepção de controle de planejamento (doze variáveis). Nesta seção são contemplados os procedimentos metodológicos adotados para satisfazer o problema de pesquisa levantado.

\section{Quadro 1 - Itens do construto Intenção Empreendedora}

\begin{tabular}{|c|c|}
\hline Dimensão & Itens \\
\hline \multirow{5}{*}{$\begin{array}{l}\text { Atitudes } \\
\text { pessoais }\end{array}$} & AT1 - Para mim, ser um empreendedor traz mais vantagens do que desvantagens; \\
\hline & AT2 - Uma carreira como empreendedor me parece atrativa; \\
\hline & $\begin{array}{l}\text { AT3 - Se eu tivesse uma oportunidade e recursos necessários eu gostaria de abrir uma } \\
\text { empresa; }\end{array}$ \\
\hline & AT4- Ser um empreendedor me traria grande satisfação; \\
\hline & AT5- Mesmo considerando outras opções, eu preferiria me tornar um empreendedor; \\
\hline \multirow{3}{*}{$\begin{array}{l}\text { Normas } \\
\text { subjetivas }\end{array}$} & NOR1 - A minha família (como reagiria a decisão de se tornar um empreendedor); \\
\hline & NOR2 - Meus amigos (como reagiria a decisão de se tornar um empreendedor); \\
\hline & $\begin{array}{l}\text { NOR3 - Meus colegas (trabalho/faculdade) (como reagiria a decisão de se tornar um } \\
\text { empreendedor); }\end{array}$ \\
\hline \multirow{12}{*}{$\begin{array}{l}\text { Percepção } \\
\text { de controle } \\
\text { de planejamento }\end{array}$} & PERC1-Iniciar uma empresa e mantê-la funcionando seria fácil para mim. \\
\hline & PERC2- Estou preparado para iniciar uma empresa que seja viável financeiramente; \\
\hline & PERC3- O processo de criação de uma nova empresa é algo que eu posso fazer; \\
\hline & $\begin{array}{l}\text { PERC4- Eu sei como preparar um projeto para criação de uma nova empresa (plano de } \\
\text { negócios, por exemplo); }\end{array}$ \\
\hline & PERC5- Conheço os detalhes práticos necessários para se iniciar uma empresa; \\
\hline & PERC6- Se eu tentasse abrir uma empresa, eu teria uma grande chance de sucesso; \\
\hline & $\begin{array}{l}\text { PERC7-Estou pronto para fazer tudo o que for necessário para me tornar um } \\
\text { empreendedor; }\end{array}$ \\
\hline & PERC8-Meu objetivo profissional é me tornar um empreendedor; \\
\hline & PERC9-Farei todos os esforços para criar e manter o meu próprio negócio; \\
\hline & PERC10-Estou decidido em criar uma empresa no futuro; \\
\hline & PERC11-Eu já pensei muito seriamente em iniciar minha própria empresa; \\
\hline & ção real de iniciar uma empresa algum dia. \\
\hline
\end{tabular}

Legenda: AT= Atitudes Pessoais; NOR= Normas Subjetivas; PERC= Percepção de Controle de Planejamento. Fonte: Liñán e Chen (2009).

As variáveis da escala de capacidade absortiva foram montadas através da escala Likert de cinco pontos, variando de "discordo totalmente" a "concordo totalmente", sendo o número 1 o ponto mais baixo (discordo totalmente) e 5 o ponto mais alto (concordo totalmente). Observa-se, no Quadro 1, que o instrumento está dividido em três grupos de afirmações, assim formadas: atitudes pessoais, normas subjetivas e percepção de controle de planejamento. A análise dos dados foi feita por estatística multivariada, por análise fatorial exploratória, por meio do Software Smart PLS® v. 3.0 (RINGLE; WENDE; BECKER, 2015)) 


\section{ANÁLISE E INTERPRETAÇÃO DOS DADOS}

\subsection{Caracterização da Amostra e dos Respondentes}

Seguindo a estruturação do modelo da Figura 1, parte-se para a especificação do modelo de mensuração que representa as relações entre os construtos e suas variáveis indicadoras correspondentes.

Uma primeira análise foi realizada em função das variáveis demográficas consideradas no estudo. Observou-se que estudantes do sexo feminino representaram $64 \%$ da amostra. Em relação à idade, 38,32\% deles possuem até 25 anos, e 36,45\% possuem de 26 a 30 anos, já os demais $26,25 \%$ possuem 31 anos ou mais. Destes $88,79 \%$ são solteiros, $9,35 \%$ casados e $1,87 \%$ divorciados. No contexto da área de ensino 54,21\% da amostra pesquisada são pós graduandos de Administração e 45, 79\% pertencem as demais áreas. A seguir apresenta-se a especificação do modelo de mensuração.

\subsection{Resultados}

O banco de dados contém 107 registros. O algoritmo do SmartPLS ${ }^{\circledR}$ foi configurado para 7 critérios de finalização. A ponderação com base no caminho foi o sistema parametrizado, pois proporciona um valor de $\mathrm{R}^{2}$ mais elevado para variáveis latentes endógenas. Foi definida 300 interações para representando o número máximo de iterações que será usado para calcular os resultados PLS. Os pesos iniciais para os indicadores externos foram definidos como 1,0. Após executar o SmartPLS ${ }^{\circledR}$ obteve um total de 140 interações. Após a definição dos parâmetros do sistema e a exclusão das variáveis PCP_01 e PCP_04 cujas cargas fatoriais foram 0,357 e 0,228 respectivamente, calculou-se o Alfa de Cronbach $(\alpha)$, a Confiabilidade Composta $\left(\rho_{c}\right)$ e a validade convergente medida pela Variância Média Extraída (AVE) do modelo de caminhos (Tabela 1).

Tabela 1 - Alfa de Cronbach, Confiabilidade Composta e Validade Convergente para o modelo de mensuração

\begin{tabular}{lccc}
\hline Dimensões & $\begin{array}{c}\text { Alfa de } \\
\text { Cronbach }(\alpha)\end{array}$ & $\begin{array}{c}\text { Confiabilidade } \\
\text { Composta }(\rho c)\end{array}$ & $\begin{array}{c}\text { Variância Média } \\
\text { Extraída (AVE) }\end{array}$ \\
\hline Atitudes Pessoais & 0,859 & 0,900 & 0,650 \\
Normas Subjetivas & 0,779 & 0,868 & 0,687 \\
Percepção de Controle de Planejamento & 0,900 & 0,914 & 0,533 \\
\hline
\end{tabular}

Fonte: Desenvolvido pelos autores: Software Smart PLS® v. 3.0 (RINGLE; WENDE; BECKER, 2015). 
Observa-se na Tabela 1 que os valores de alfa de Cronbach e confiabilidade composta possuem valores de 0,7 a 0,90 considerados bons e eficientes, quanto a $A V E$, os valores obtidos são superiores à 0,5, não sendo necessário a remoção de cargas fatoriais, concluindo-se confiabilidade composta do modelo. A Tabela 2 apresenta os valores das cargas fatoriais cruzadas das variáveis observadas nas variáveis latentes.

Tabela 2 - Cargas fatoriais cruzadas das variáveis observadas nas variáveis latentes para a mensuração

\begin{tabular}{lccc}
\hline \multirow{2}{*}{ Indicadores } & \multicolumn{3}{c}{ Variáveis Latentes (Dimensões) } \\
\cline { 2 - 4 } & Atitudes Pessoais & Normas Subjetivas & Percepção de Controle de Planejamento \\
\hline AP_01 & 0,525 & 0,167 & 0,290 \\
AP_02 & 0,868 & 0,117 & 0,652 \\
AP_03 & 0,797 & 0,062 & 0,624 \\
AP_04 & 0,921 & 0,153 & 0,732 \\
AP_05 & 0,858 & 0,149 & 0,740 \\
NS_01 & 0,158 & 0,845 & 0,098 \\
NS_02 & 0,114 & 0,824 & 0,100 \\
NS_03 & 0,094 & 0,817 & 0,056 \\
PCP_02 & 0,492 & 0,027 & 0,650 \\
PCP_03 & 0,238 & 0,151 & 0,450 \\
PCP_05 & 0,069 & 0,103 & 0,360 \\
PCP_06 & 0,261 & 0,188 & 0,522 \\
PCP_07 & 0,631 & 0,031 & 0,858 \\
PCP_08 & 0,787 & 0,155 & 0,838 \\
PCP_09 & 0,607 & 0,114 & 0,776 \\
PCP_10 & 0,651 & 0,028 & 0,891 \\
PCP_11 & 0,691 & 0,074 & 0,838 \\
PCP_12 & 0,715 & 0,064 & 0,876 \\
\hline Fon_
\end{tabular}

Fonte: Desenvolvido pelos autores: Software Smart PLS® V. 3.0 (RINGLE; WENDE; BECKER, 2015).

A análise da validade discriminante, apresentada na Tabela 3, possibilita verificar de que as cargas fatoriais das variáveis observadas (indicadores) nas dimensões (variáveis latentes) originais são maiores que em outros, constatando-se que há validade discriminante por este critério. Para isso, utiliza-se o critério Fornell-Larcker que compara a raiz quadrada das AVE's com as correlações das variáveis latentes. Este critério baseia-se na ideia de que uma dimensão partilha mais variância com seus indicadores observáveis associados do que com qualquer outra dimensão (Tabela 3). 
Tabela 3 - Análise da validade discriminante pelo critérios Fornell-Larcker e HTMT para o modelo de mensuração

\begin{tabular}{|c|c|c|c|}
\hline Dimensões & AP & NS & PCP \\
\hline & \multicolumn{3}{|c|}{ Critério Fornell-Larcker } \\
\hline Atitudes Pessoais & 0,806 & & \\
\hline Normas Subjetivas & 0,154 & 0,829 & \\
\hline \multirow[t]{2}{*}{ Percepção de Controle de Planejamento } & 0,784 & 0,106 & 0,730 \\
\hline & \multicolumn{3}{|c|}{ Critério Heterotrait-Monotrait Rati } \\
\hline \multicolumn{4}{|l|}{ Atitudes Pessoais } \\
\hline Normas Subjetivas & 0,190 & & \\
\hline Percepção de Controle de Planejamento & 0,786 & 0,153 & \\
\hline
\end{tabular}

De acordo com a Tabela 3, o modelo de mensuração, atende ao critério FornellLarcker uma vez que os valores da raiz quadrada das AVE's dos construtos (diagonal principal) são superiores aos valores das correlações entre estes. Quanto ao critério HTMT (Heterotrait-Monotrait Ratio), pode-se verificar que todos os pares de dimensões ficam abaixo de 0,90, atendendo aos preceitos de Henseler, Ringle e Sarstedt (2019).

Para testar se os valores de $\beta$ 's são significativamente diferentes de zero, utilizase da opção bootstrapping (5.000 subamostras) para a obtenção e verificação considerando os intervalos de confiança e os valores do teste $t$ a fim de confirmar ou não as hipóteses $\mathrm{H}_{1}, \mathrm{H}_{2}$ e $\mathrm{H}_{3}$ (HAIR JR. et al., 2014) (Tabela 4).

Tabela 4 - Intervalo de confiança para os $\beta^{\prime}$ s para 5.000 subamostras para o modelo estrutural

\begin{tabular}{|c|c|c|c|c|c|c|}
\hline Hipótese Dimensão & $A r$ & & Intervalo de & ança (IC) & & \\
\hline$\rightarrow$ Dimensão & ra $U T_{3}$ & IVIE & $2,5 \%$ & $97,5 \%$ & & p-valc \\
\hline $\mathrm{H}_{1}: \mathrm{AP} \rightarrow \mathrm{PCP}$ & 0,786 & 0,792 & 0,727 & 0,847 & 25,785 & 0,000 \\
\hline $\mathrm{H}_{2}: \mathrm{NS} \rightarrow \mathrm{PCP}$ & $-0,014$ & $-0,009$ & $-0,131$ & 0,111 & 0,233 & 0,816 \\
\hline $\mathrm{H}_{3}: \mathrm{NS} \rightarrow \mathrm{AP}$ & 0,154 & 0,182 & $-0,055$ & 0,353 & 1,638 & 0,101 \\
\hline
\end{tabular}

Fonte: Desenvolvido pelos autores: Software Smart PLS® v. 3.0 (RINGLE; WENDE; BECKER, 2015).

Na Tabela 4, a coluna "amostra original (O)" mostra os valores originais dos $\beta$ 's para cada combinação das dimensões, a coluna "média da amostra (M)" apresenta os valores médios de HTMT para as 5.000 amostras pelo método de bootstrap. As colunas "2,5\%" e "97,5\% mostram o limite inferior e superior do intervalo de confiança. Observou-se que conforme Henseler, Ringle e Sarstedt (2015) o valor de $\beta$ 's para o intervalo de confiança definido ficou abaixo de 0,90 conforme propõe atestando a 
validade discriminante, ou seja, as medidas dos construtos do modelo não se correlacionam com outros construtos dos quais se supõe que devam divergir.

Quanto aos valores do teste t, ou seja, a relação entre os constructos e os valores de b, para que o Beta seja aceito, deve-se testar a relação causal entre os dois constructos e verificar se é significante ou não. Assim, utilizou-se o teste t de Student, em que valores acima de 1,96 são considerados significantes a 5\%, isto é, os constructos são relacionados (HAIR et al., 2014).

Conforme observado na tabela anterior, o teste $t$ apresenta-se significante observa-se que somente a hipótese $\mathrm{H}_{1}$ confirmou a hipótese de caminho $\left(\mathrm{H}_{0}: \Gamma=0\right.$ coeficientes de caminho $=0$ ), $p<0,05$, ou seja, a atitude pessoal se relaciona direta e positivamente com a percepção de controle de planejamento. As demais hipóteses $\mathrm{H}_{2}$ e $\mathrm{H}_{3}$ foram rejeitadas, $\mathrm{p}>0,05$, ou seja, as normas subjetivas se não se relacionam direta e positivamente com a percepção de controle e planejamento não se relacionam direta e positivamente com as atitudes pessoais.

Para avaliar a qualidade do modelo estrutural pelo PLS-SEM as medidas mais importantes são o $\mathrm{R}^{2}$ (coeficiente de explicação), $f^{2}$ (tamanho do efeito), Q² (relevância preditiva) e a significância dos coeficientes do caminho estrutural.

A Tabela 5 apresenta os valores de $R^{2}$ e $R_{\text {ajustado }}^{2}$ para o modelo estrutural.

Tabela 5 - Coeficiente de determinação R2 e $\mathrm{R}_{\text {ajustado }}^{2}$ para o modelo estrutural e suas significâncias

\begin{tabular}{lll}
\hline Dimensões & $\mathrm{R}^{2}(p-$ valor $)$ & $R_{\text {ajustado }}^{2}(p$-valor $)$ \\
\hline Atitudes Pessoais & $0,024(0,474)$ & $0,014(0,667)$ \\
Percepção de Controle de Planejamento & $0,615(0,000)$ & $0,607(0,000)$ \\
\hline
\end{tabular}

Fonte: Desenvolvido pelos autores: Software Smart PLS® V. 3.0 (RINGLE; WENDE; BECKER, 2015).

Observa-se na Tabela 5 que a dimensão Atitude Pessoais (AP) $\left(R^{2}=0,024\right)$ possui um efeito fraco e não significativo já a Percepção de Controle e Planejamento (PCP) ( $R^{2}$ $=0,615)$, apresenta coeficiente de explicação com efeito forte e significativo, pois segundo Wetzels et al. (2009) valores acima de 0,36 são considerados adequados para pesquisas nas áreas de ciências sociais.

A medida de tamanho do efeito $f^{2}$ é utilizada para avaliar se haverá impacto substancial nos construtos endógenos se forem omitidos construtos (HAIR JR. et al., 
2014). O tamanho do efeito ( $\left(^{2}\right)$ ou indicador de Cohen, é o valor obtido pela inclusão e exclusão de constructos do modelo (um a um), valores entre 0,02 e 0,15 são considerados pequenos, valores entre 0,15 e 0,35 são considerados médios e valores acima de 0,35 são considerados grandes (HAIR JR. et al., 2014). Ringle, Silva e Bido (2014, p. 70) avaliaram quanto o construto é útil para o modelo (Tabela 6). A Tabela 6 apresenta os valores do tamanho do efeito $f^{2}$ para o modelo estrutural, sendo que as colunas apresentam as dimensões endógenas e as linhas apresentam as correspondentes dimensões exógenos.

Tabela 6 - Tamanho do efeito f2 para o modelo estrutural e suas significâncias (p-valor)

\begin{tabular}{lcc}
\hline Dimensões & \multicolumn{2}{c}{ Dimensões Endógenas } \\
\cline { 2 - 3 } Exógenas & Atitudes Pessoais & Normas Subjetivas \\
\hline AP & & $1.566(0,000)$ \\
NS & $0,024(0,525)$ & $0,001(0,971)$ \\
PCP & & \\
\hline
\end{tabular}

Fonte: Desenvolvido pelos autores: Software Smart PLS® v. 3.0 (RINGLE; WENDE; BECKER, 2015).

Na Tabela 6, o tamanho do efeito dos construtos, verificou-se o tamanho do efeito $f^{2}$ ou indicador de Cohen, valor que é obtido pela inclusão e exclusão de constructos do modelo (um a um), valores entre 0,02 a 0,15 são considerados pequenos, valores entre 0,15 a 0,35 são considerados médios e valores acima de 0,35 são considerados grandes (HAIR et al., 2014). A dimensão exógena AP sobre a dimensão endógena PCP $(1,566 ; 0,000)$ é classificada como grande. Já o tamanho do efeito da dimensão NS sobre a dimensão endógena $\operatorname{AP}(0,024)$ e NS sobre $\operatorname{PCP}(0,001)$ são classificados como pequenos e não significativos.

A medida de validade preditiva $\mathrm{Q}^{2}$, também conhecido por indicador de StoneGeisser, avalia a acurácia do modelo ajustado. De acordo com Hair Jr. et al. (2014), no modelo estrutural valores de $\mathrm{Q}^{2}$ maiores que zero indicam a relevância preditiva do modelo de caminhos. Os valores de $\mathrm{Q}^{2}$ estimados pelo processo blindfolding representam uma medida de quão bem o modelo de caminho pode prever os valores originalmente observados. A Tabela 7 apresenta os valores da medida de relevância preditiva $\mathrm{Q}^{2}$ para o modelo estrutural. 
Tabela 7 - Valores Q2 para o modelo estrutural

\begin{tabular}{llll}
\hline Variáveis Latentes & SSO & SSE & $\mathrm{Q}^{2}=1-\frac{\text { SSE }}{\text { SSO }}$ \\
\hline Atitudes Pessoais & 535,000 & 528,959 & 0,011 \\
Normas Subjetivas & 321,000 & 321,000 & --- \\
Percepção de Controle de Planejamento & $1.070,000$ & 768,375 & 0,282 \\
\hline SSO = Soma dos Quadrados Observados; SSE = Soma dos Quadrados dos Erros Estimados \\
Fonte: Desenvolvido pelos autores: Software Smart PLS® V. 3.0 (RINGLE; WENDE; BECKER, 2015).
\end{tabular}

Na Tabela 7 apresentam-se os valores de $\mathrm{Q}^{2}$ com base nos resultados totais do procedimento blindfolding, sendo que na coluna SSO (Sum of the Square do Observations) mostra-se a soma dos quadrados observados, na coluna SSE (Sum o the Squared Prediction Errors) a soma dos quadrados dos erros de estimação e a última coluna o valor final de $\mathrm{Q}^{2}$, isto é, a relevância preditiva do modelo no que diz respeito a cada construção endógena. Com base nos resultados, observa-se que o modelo é relevante, visto que os valores de $\mathrm{Q}^{2}$ são maiores que zero. O diagrama de caminhos final para as equações estruturais apresenta-se na Figura 2.

Figura 2 - Diagrama de caminhos final para equações estruturais do modelo estrutural

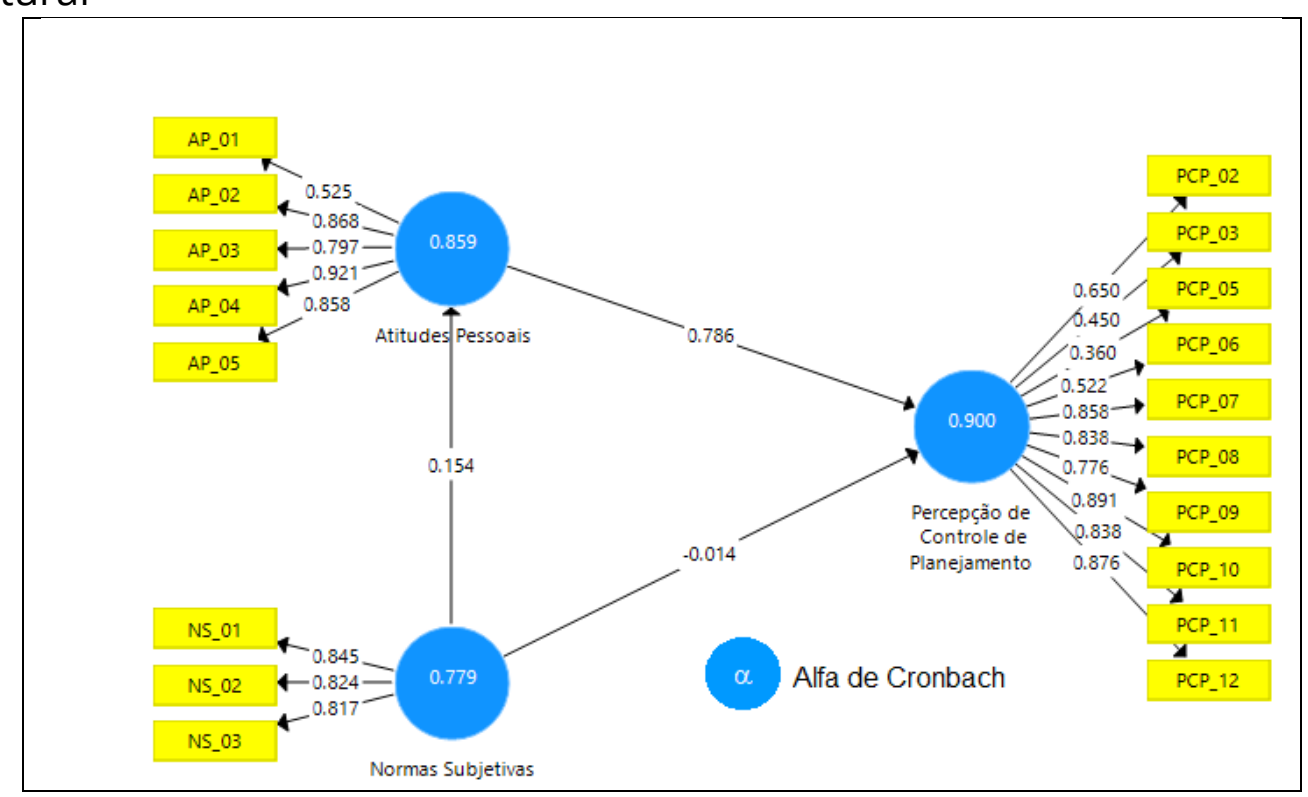

Fonte: Desenvolvido pelos autores (Software Smart PLS® V. 3.0 (RINGLE; WENDE; BECKER, 2015)).

A partir desses construtos, foram testadas as três hipóteses delineadas nesse estudo. Como resultado, obteve-se que as hipóteses $\mathrm{H}_{2}$ e $\mathrm{H}_{3}$ foram rejeitadas, enquanto que $\mathrm{H}_{1}$ (Atitudes Pessoais se relacionam direta e positivamente com a Percepção de Controle de Planejamento) foi confirmada (Tabela 4) uma vez que o 
coeficiente foi estatisticamente significante, apresentando valores de teste $t$ acima do nível de significância de 5\%. Quanto a mensurar os efeitos de cada variável e identificar as que possuem maior relevância para o modelo, foi observada que a variável mais explicada corresponde ao Controle do Comportamento Planejado $\left(R^{2}=0,615\right.$ e $Q^{2}=$ 0,282 ) os demais coeficientes de explicação foram considerados não significativos ( $p>$ $0,05)$.

\section{CONSIDERAÇÕES FINAIS}

O objetivo da pesquisa foi discutir as dimensões da intenção empreendedora (atitudes pessoais, normas subjetivas e percepção de controle de planejamento), adotando como análise o comportamento de alunos de pós graduação. Utilizou-se como base teórica a perspectiva de Liñán e Chen (2009), uma vez que estes autores apontam as três dimensões acima citadas. O artigo em questão obteve êxito ao conseguir elucidar o posicionamento de estudantes de pós graduação frente a intenção em empreender e como suas variáveis se relacionam com a Intenção Empreendedora.

O problema da pesquisa e suas devidas hipóteses foram respondidos com quais variáveis influenciam a intenção empreendedora através de equações estruturais utilizando o software Smart PLS ${ }^{\circledR}$ v. 3.0 (RINGLE; WENDE; BECKER, 2015) para validar o modelo de acordo com alguns indicadores como: alfa de cronbach, confiabilidade de item, validade discriminante, colinearidade estatística e variância extraída média (AVE). As hipóteses H2 $(-0,014)$ = Normas Subjetivas não se relacionam direta e positivamente com a Percepção de Controle de Planejamento e H3 $(0,154)=$ Normas Subjetivas não se relacionam direta e positivamente com as Atitudes Pessoais foram rejeitadas.

Foi aceita somente a hipótese H1 $(0,786)=$ Atitudes Pessoais se relacionam direta e positivamente com a Percepção de Controle de Planejamento. Essa pesquisa ajudará a compreender o que leva um estudante de pós graduação a empreender, já que sabemos as variáveis que mais influenciam essa decisão. 


\section{REFERÊNCIAS}

AJZEN I. From intensions to actions: A theory of planned behavior. In J. Khul \& J. Beckman (Eds.), Action-control: From cognition to behavior, 11-39, 1985.

AJZEN I. The theory of planned behavior. Organizational Behavior and Human Decision Processes, 50: 179-211, 1991.

APPOLINÁRIO F. Dicionário de metodologia científica. 2. ed. São Paulo: Atlas, 2011.

ARAÚJO CMB. Intenção empreendedora dos alunos de graduação em administração da UNB: como a trajetória no curso os afeta? Brasília, 2014.

BIRD B. Implementing entrepreneurial ideas: The case for intentions. Academy of Management Review, 13(3): 442-454, 1988.

CARTER NM, GARTNER WB, REYNOLDS PD. Exploring start-up event sequences. Journal of Business Venturing, 11(3): 151-166, 1996.

DAVIDSSON P. Determinants of entrepreneurial intentions. Comunicação apresentada na conferência Rent IX, Piacenza, Itália, 23-24 de novembro, 1995.

FISHBEIN MA, AJZEN I. Belief, attitude, intention and behavior: an introduction to theory and research. Reading: Addison-Wesley, 1975.

GIL AC. Como elaborar projetos de pesquisa. São Paulo: Atlas, 1996.

GIL AC. Como elaborar projetos de pesquisa. 5. ed. São Paulo: Atlas, 2010.

HAIR Jr. JF, HULT TM, RINGLE CM, SARSTEDT M. Primer on Partial Least Squares Structural Equation Modeling (PLS-SEM). Sage, 2014.

HANDRIMURTJAHJO AD, SETIADI NJ, KUNCORO E. The Role of Entrepreneurship Education in Forming Students' Entrepreneurial Attitudes. Advanced science letters, 21(4), 2015.

HENSELER J, RINGLE CM, SINKOVICS RR. The use of partial least squares path modeling in international marketing. Advances in Internation Marketing (AIM), 20: 277-320, 2009.

LIÑÁN F, CHEN YW. Development and Cross-Cultural application of a specific instrument to measure entrepreneurial intentions. Entrepreneurship Theory and Practice, 33(3): 593-617, 2009. 
LIÑÁN F, URBANO D, GUERRERO M, Regional variations in entrepreneurial cognitions: start-up intentions of university students in Spain. Entrepreneurship \& Regional Development, 23(3-4): 187-215, 2011.

LOIOLA E. et Al. Ação planejada e intenção empreendedora entre universitários: analisando preditores e mediadores. Rev. Psicol., Organ. Trab., Brasília, 16(1): 22-35, 2016.

MUELLER J, ZAPKAU FB, SCHWENS C. Impact of prior entrepreneurial exposure on entrepreneurial intention - cross-cultural evidence. Journal of Enterprising Culture, 22(3): 251-282, 2014.

RINGLE CM, SILVA D, BIDO D. Modelagem de equações estruturais com utilização do SmartPLS. Brazilian Journal of Marketink, 13(2), 2014.

RINGLE CM, WENDE S, BECKER JM. SmartPLS 3. Bönningstedt: SmartPLS, 2015.

WETZELS M, ODEKERKEN-SCHRODER G, VAN OPPEN C. Using PLS path modeling for assessing hierarchial construct models: guidelines and empirical illustration. MIS Quarterly, 33(1): 177-195, 2009. 